\title{
Brainstorming Versus Arguments Structuring in Online Forums
}

\author{
Abdulrahman Alqahtani \\ Department of Computer Science \\ Najran University \\ Najran, Saudi Arabia
}

\author{
Marius Silaghi \\ Department of Computer Science \\ Florida Institute of Technology \\ Melbourne, FL, USA
}

\begin{abstract}
We characterize electronic discussion forums as being of one of the following two types: Brainstorming Forums and Arguments Structuring Forums. In this work we analyze and classify the types of threading models occurring as a function of the type of forum. For our analysis we study forums attached to the 25 news sources most used by the aggregator Google News, as detected by a 2007 study. Most discussion forums associated with articles on these news sources seem to be designed not with the purpose of structuring arguments but mainly with the purpose of helping readers brainstorm easily their reactions to the corresponding news item. The forums were classified as to what user-supported metadata they gather and use in comment presentation.

We compare the features observed for brainstorming forums, as learned via the aforementioned procedure, with the features of dedicated argument structuring forums. The argument structuring forums that were used as basis of the comparison are: YourView, DebateDecide, and Opinion Space. We notice significant differences in the obtained models for the two types of forums, as well as significant differences with respect to the the structuring of user submitted data in polls associated with major news channels.
\end{abstract}

We believe that this is the first kind of work that deals with the issue above.

Keywords: Knowledge Representation, Threading Models for Arguments in Electronic Debates, Threading Model Classification, Debate Threading Model, Comparison Online news Platforms

\section{INTRODUCTION}

Even before Internet users were heavily engaged in social networks like Facebook and Twitter, many of them were attracted by social applications, such as commented news, that drew significant participation and activism in the early 2000s [1].

Various other applications of electronic debates emerged in the same period of time, such as product reviews and evaluation used for collaborative filtering on online stores like Amazon or Recommendation sites like Yelp [2].

While these may be some of the most traditionally used applications of debates, a large number of other applications appeared in varied domains such as: political, religious, science, and education reviews [3].

Various foundations fund open software development for supporting comments and debates to be associated to blogs and online news channels, as a way to promoting civil engagement and a civil society [4].

Given the effort spent by developers and foundations to build electronic debate mechanisms, the question arises on whether one platform fits it all, or platforms have to be adjusted to the actual domain [5].

Here we compare properties and expectations user have from two different and well established domains of application for electronic debates. In particular, we investigate the expectations and behavior of users with respect to brainstorming of comments, and argument structuring. The results of this study is used to detect relevant differences between the studied domains.

\section{BACKGROUND}

Open electronic debate platforms can be used with application domains, from politics to entertainment, e.g. Slashdot [6]. News articles or product descriptions can each be associated with a forum. Some studies have focused on the impact of online comments as being open news by themselves [6]. We address the issue of management for such open electronic debate platforms. Namely, of the impact of their organization (e.g., threading model) on the quality of user experience (relevance, redundancy, diversity, importance, clarity, efficiency) [7], [8], [9], [10]. We are investigating the user expectation and beliefs concerning platforms for comments concerning products evaluations and news articles [10].

Understanding of the given problem is improved as the user gets acquaintance with the relevant justification provided by other participants. An essential ingredient comes from the correct evaluation of the importance of a justification as yielded by the number of participants supporting it. Another important factor in catalyzing the understanding of a justification is the intensity with which each participant supports that justification. In electronic debates, users can support somebody else's justification as an alternative to providing his/her own justification. Justifications with large support can be favored by viewers, as they may better represent the opinion of the group. A further mechanism to help users locate relevant justifications is based on threading. Namely, new justifications can point to old justifications that they claim to refute or enhance. Thereby people visualizing old justifications are notified of 
the presence of the refutation and enhancement claims. In a DDP2P application, all debates and arguments with news have to be related to a motion in a given organization. The user can vote on any with only one justification and he/she can post news linked to motions or justifications [11], [12], [13].

Namely, if a majority of peer members disagree with a motion that the user has earlier believed to be good, he may reconsider his position on the motion. The peers could have potentially discovered problems with that motion, problems communicated via justifications that can make the constituent withdraw his/her support. Withdrawing support for an unpopular motion will save the time of the other constituents who will be less tempted to spend time reading it, and this will help the organization to save the resources needed to move on the proposal and organize an official ballot [12], [13].

\section{Related Research AREA}

A few researchers have already addressed the relationship between social network behavior and these networks' intended purposes [14], [15]. This section highlights previous studies on the type and quality of collaboration based on social networks. In this chapter, we provide a review of the literature related to the work described in this dissertation. It also presents the gaps in the existing research literature. In this research, the related work is presented and classified according to the means and purpose of the studied collaboration.

\section{A. Generic Tools for Collaboration}

We identify the following types of generic tools for collaboration, on an idea, between unsophisticated users:

- Arguments

- Thumb up/down

- Voting

- Threading

a) Arguments: According to the Merriam Webster dictionary, argumentation is "the act or process of giving reasons for or against something: the act or process of making and presenting arguments" where an argument is "a statement or series of statements for or against something" [16]. The possibility to express arguments in favor of or in opposition to ideas is considered an important instrument in collaboration [17].

b) Thumb up/down: An easy way to garner feedback on ideas and arguments is to let people show their opinion using simple tags such as a thumbs-up or a thumbs-down [18]. Often this is done in systems where participants are not authenticated. Moreover, users can often use a thumbs-up tag for opposing arguments, thereby making it difficult to use these tags for extracting reliable statistics.

c) Voting: Voting is an approach to measure the opinion of a certain group of people. Unlike the thumbs-up/thumbsdown practice mentioned above, commonly the identity of people involved in voting is verified and each person can vote only once. Therefore, a user cannot vote simultaneously on opposing arguments [19]. Statistics about opinions can be reliably estimated [20]. d) Threading: Many writers are skeptical about the usefulness of the comments or threads that are placed on common web forums [21], despite the large number of useful comments. However, the idea of abolishing comments is not popular. Users feel more motivated to visit forums where they can comment since they may believe in the impact of these comments [22]. Besides collaborating on ideas, people also collaborate on projects by contributing work or resources [23].

\section{B. Discourse Architecture}

Discourse architecture is the technique to provide environments for users to connect with each other through networked computers. Discourse architecture is supporting the discussions, conversions, debates and arguments between users in computer social media or networks [24], [25].

\section{Major Knowledge Representation Types}

\section{Classification:}

\section{A. Ontologies}

The research in Ontologies deals with the establishment of languages that have no ambiguities. The language should be powerful enough to represent knowledge. For example, a message based on a standard ontology starts by a reference to the ontology version, and then has to follow its strict syntax [26], [27]. Ontologies define communities.

\section{B. Logic}

One of the techniques, that is used to store sentences within the computer, is mathematical logic. It is used in knowledge databases such as those used by PROLOG and GOLOG [28], [29]. They are difficult to use with general users [30]. Also, some applications such as ArgTrust that build to help the user to get information source by using logical argumentation and a computational model of trust.

\section{Semantic Networks}

A Semantic Network consists of a set of nodes that are linked by arcs. In general, nodes represent concepts while arcs represent the relations between concepts. There are exited researches for analysis the language by studying the nature language and its relevant with meaning, grammars, lexis and societies [31], [32].

\section{Mind Maps}

Mind Map is the name given to a tool or a means of expressing human ideas. It is drawn on a forked tree and relies on the pictures and words in the human memory. Since the human memory is a graphic memory, which depends on information linked with pictures, mind maps help to save and retrieve information easily [33]. The use of mind maps is an innovative way to assist students to understand key information. Mind maps have the ability to provide students with an information retention strategy, integration of critical thinking, and problem solving skills [34]. 


\section{E. WordNet}

WordNet is a huge lexical database of English [35]. It is a set of cognitive synonyms that have been collected from nouns, verbs, adjectives, and adverbs with a clear expression of concept [36].

\section{F. Argument Maps}

An Argument Map is a logic structure for arguments. It breaks up the argument into statement, reason, or fact. Also, it shows the relation between parts of the argument [37]. A lot of people have difficulty understanding complex arguments presented in textual form. An argument map is a graphical way to represent the complex relations between relevant statements. Therefore, it can improve human comprehension. It looks similar to a flowchart and a specialization/generalization hierarchy [38]. There exist tools that can be used to build argument maps [39]. These argument maps have been built to help people better understand the issues related to a given problem. Arguments are frequently attached to emotions. In argument maps, there is no requirement for arguments to be objective. Simple and complex arguments are captured with the help of the diagram [37].

\section{Evaluation of KNOWledge RePresentation MECHANISMS}

Here I present some techniques that can be used to evaluate knowledge representation:

\section{A. Surveys}

Surveys can be used for knowledge representation evaluation [40], [41]. In 2009, McDonald et al evaluated three formats of privacy policies for six companies. There were 749 internet users for this study and each filled out the survey to evaluate a company's privacy with one format [42].

In 2005, Janez Brank presented a survey of the state of the art in ontology evaluation [43]. He grouped the ontology evaluation approaches depending on level of evaluation, as follows:

- Lexical, vocabulary, or data layer:

This level focused on concepts, instances, facts, etc, which are included in the ontology. Also it considers the vocabulary that is used to represent these concepts. Evaluation in this level involves comparison with different sources of data.

- Hierarchy or taxonomy: It studies the relation between concepts

- Other semantic relations:

This level includes other relations between concepts besides is-a. Evaluation of this level contains measures such as precision and recall.

- Context or application level:

The Context level occurs when an ontology becomes part of a huge collection of ontologies, which is mentioned by separate definitions in those other ontologies. Evaluation at this level may help to take the context into account while evaluating different definitions. application is another form of context where the ontology is to be used.

- Syntactic level:

The Syntactic level matches the syntactic requirements of the particular formal languages that describe the ontology.

- Structure, architecture, design:

This is primarily manually constructed ontologies that evaluate certain pre-defined design principles or criteria for further development.

\section{B. Case Studies}

A few case studies exist that have been to improve the knowledge representation evaluation [44], [45]. In 2003, Kim and Chan proposed a way to evaluate reordered Google search results by associating each item with a score provided by clusters of human users and aggregating the total score based on the position of the valuable items [46].

\section{Panels}

The panel (group of people) can be used to compare different forms of knowledge representation [47], [48], [41]. Bobrow, in his research (a panel on knowledge representation), mentions that he asked panel members to briefly answer three questions in order to compare different forms of knowledge representation [48]. The three panel questions were: "What are the most important premises underlying your approach to knowledge representation, the critical ideas, and major mechanisms used in your system [48]".

\section{Simulations}

Simulation is also a tool that helps to evaluate knowledge representation to improve quality and shorten time-tocompare [49], [50]. Zhou and his team provide simulation modeling based on knowledge representation. They presented a notation to formalize the representations to support their model development and validation and to facilitate the analysis and translation of the representations [50]. In this study, they discussed solving complex problems, such as cost, quality, productivity, and shortening time-to-market by using a knowledge engineering approach [50].

There are many benefits of formal Knowledge Representation Models [51]. Some mechanisms are intended to simplify automatic data mining [52]. Data Mining is basically a process for inspecting and searching for specific information in large amounts of data. Other knowledge representation mechanisms are used to enhance human comprehension, such as mind maps, and argument maps [51]. To evaluate arguments, different techniques are discussed in [53]. Graphical representation of evidence-based dialogue, questions, ideas, pros, cons, and data helps in scientific reasoning [54]. In teaching, argumentation mapping helps the student to build critical thinking skills. 


\section{Threading Models for Arguments IN ELECTRONIC DEBATES}

\section{A. Introduction to Threading Models}

The relevant point to mention about threading models is that users can benefit in their decision making by exploring comments and justifications provided by others (e.g., on fora or various social networks) [55]. This exploration can be enhanced not only by search engines, but also by using hints provided by links generated by the comment authors themselves [55].

The whole World Wide Web was designed for improving information exploration based on links (here hyperlinks) [56]. The Internet fora and social networks took this a step further by hosting such links in a more compact way but typically at the expense of generality. Namely restrictions are added as to how many links are possible from each article item [57]. The most common and simple restriction is one where each article (comment) can link to a single other article, to which it is a "reply". This structure, together with restrictions on the maximum chain of "replies", gives rise to the so-called tree structures seen on common Internet fora. A compact representation can be enabled by such rules [58].

Conversation Threading has been standardized for the IMAP email systems [59]. A common technical classification is:

- Client based: Microsoft Outlook, Thunderbird [60]

- Web based: Gmail, Reddit, Slashdot [7]

\section{B. Examples of Threaded Fora in Online Forums}

There are many fora on the Internet. They are frequently attached to articles in online newspapers or next to items in online shops or product rating sites. Here we focus on fora that are specially designed to support debates on online news. Some of the other fora are occasionally used for debates around linked issues but they are not generally designed to help navigate or extract a conclusion for those debates.

The 24 fora that we have identified as specially designed for Online News are [61]:

- New York Times

- Washington Post

- Houston Chronicle

- Bloomberg

- Los Angeles Times

- Reuters

- Forbes

- Monsters and Critics.com

- Guardian Unlimited

- Voice of America

- International Herald Tribune

- Boston Globe

- Chicago Tribune

- BBC News

- San Francisco Chronicle

- CBS News

- Times Online
- Wall Street Journal

- USA Today

- FOX News

- $\mathrm{CNN}$

- Seattle Post Intelligencer

- MSNBC

- ABC News

All above forums are online news platforms for presenting comments and helping users to explore these comments by scoring them. In most of these platforms, registered users have the ability to submit multiple comments or replies. Moreover, the participant can give a thumbs up or thumbs down for comment.

One can observe that online news platforms are significantly different from newspapers. An effort was made over the centuries to find optimal ways of organizing face-to-face debates [62]. Research is needed to decide whether current online news platforms are carried out with the optimal debate threading models and debate representation, as a means of knowledge representation.

The set of restrictions placed on the number and type of links in an article item, as well as on the global rules on types and size of link chains, form a "debate threading model."

For a given threading model, there are multiple ways of graphically displaying the result to users. For example, some items (i.e., at given depths in the tree) could be hidden until required by users. Otherwise, links can be shown graphically or the number or existence of connected items can just be mentioned. We used to separate the knowledge relations by their representation.

The set of rules describing how debate instances are graphically presented to viewers is called "debate representation model."

Some fora specialize on debates for different topics: music, fashion, software bugs, and politics. Since some topics are more disputable than others, different threading models may be appropriate for different topics and, thus, fora.

\section{Comparison The Sources Of Google News}

This study focuses on The Sources Of Google News structured. According to Google News Report USA, the top 25 news sources year to day as of May 30, 2007 [61], we can compare these fora across six criteria to better understand differences. Table I compares The Sources Of Google News.

a) Criteria:

- Voting: It is a score that counts the number of supports. This criterion has as domain:

- None

- Report with reasons

- Report as abusive

- Report with corrections 


\begin{tabular}{|c|c|c|c|c|c|}
\hline & Voting & Comments & Threads & Emotion & Metrics of ordering \\
\hline New York Times & None & Not classified & $\overline{\text { Reply to }}$ & Recommended/thump up & By Newest and Oldest, \\
\hline Washington Post & None & Not classified & Reply & Like & By Newest, Oldest, Most replies or most liked \\
\hline Houston Chronicle & None & Not classified & Submit & Like and Dislike & By Newest, Oldest, Most replies, Editor's Pick, Most active or Most liked \\
\hline Bloomberg & Report With Reason & Not classified & Reply & Recommendations & By Newest, Oldest, Recommendations, Threads Expanded/Collapsed/Unthreaded \\
\hline Los Angeles Times & None & Not classified & Reply & Like and Dislike & By Newest, Oldest, Most replies, Editor's Pick, Most active or Most liked \\
\hline Reuters & Report As abusive & Not classified & Reply & None & By Date \\
\hline Forbes & Report Corrections & Not classified & Post comment & Top comment & By Date and Top comments \\
\hline Monsters and Critics.com & None & Not classified & Reply & Best comment & By Newest, Oldest, and Best comments \\
\hline Guardian Unlimited & Report With Reason & Not classified & Reply & Recommendations & By Newest, Oldest, Recommendations, Threads Expanded/Collapsed/Unthreaded \\
\hline Voice of America & None & Not classified & Reply & None & By date, Latest First and Chronological \\
\hline International Herald Tribune & None & Not classified & Reply & None & By Newest, Oldest, and Top comment \\
\hline Boston Globe & None & Not classified & Reply & None & By Newest, Oldest, and Best comment \\
\hline Chicago Tribune & None & Not classified & Reply & Like & By Newest, Oldest, Most replies, Editor's Pick, Most active or Most liked \\
\hline BBC News & Report & Not classified & Reply & Like and Dislike & By Oldest first, Highest rated, Lowest rated \\
\hline San Francisco Chronicle & None & Not classified & Reply & Like & By Newest, Oldest, Most replies, Editor's Pick, Most active or Most liked \\
\hline CBS News & None & Not classified & Reply & like & By Newest, Oldest, and Top comment \\
\hline Times Online & None & Not classified & Submit & None & By Date \\
\hline Wall Street Journal & None & Not classified & Sign to comment & None & By Newest, Oldest, and Reader Recommended \\
\hline USA Today & None & Not classified & Reply & Like & By Newest, Oldest, and Top \\
\hline FOX News & None & Not classified & Reply & Like and Dislike & By Newest, Oldest, Popular, or Most Discussed \\
\hline CNN & None & Not classified & Reply & Like & By Newest, Oldest, or \\
\hline Seattle Post Intelligencer & None & Not classified & Reply & Like and Dislike & By Newest, Oldest, Popular, or Most Discussed \\
\hline MSNBC & None & Classified by groups & Reply & Like & By Date \\
\hline ABC News & None & Not classified & Reply & Like, Dislike and Recommended & By Date \\
\hline
\end{tabular}

TABLE I: Comparison The Sources Of Google News

- Comments/Justifications:

These are arguments presented by users. The domain of this criteria is:

- Not Classified

- Classified by groups

All study platforms provide a structured comment section. All comments are in the same column and the user once again has to go through each of them to assess the decision behind each comment. Only MSNBC provides a structured comment/justification section that is organized by group choice.

- Threads:

Threads are graphs/trees of arguments and relations between those arguments. The domain of this criteria is:

- Reply to

- Submit

- Post

- Sign

- Emotion Thumb (Up/Down):

This is a feature that allows the users to express their emotional support of an argument by selecting "Up" when they agree and "Down" for disagreement. Some platforms do not offer this feature while others have it available. The domain for this criteria is:

- Like

- Dislike

- Top

- Best

- Recommended

- None

Most of the study platforms have a feature to allow the users to express their emotion (Thumbs Up/Down).

- Metrics of Ordering:

This is a sorting option that allows the user to order the comments and arguments. The domain for this criteria is:

- Ordering by date

- Ordering by Oldest

- Ordering by Newest
- Ordering by most replies

- Ordering by most liked

- Ordering by most active

- Ordering by editor's Pick

- Ordering by Recommendations

- Ordering by Threads Extended/Collapsed/Unthreaded

- Ordering by Chronological

- Ordering by Top comments

- Ordering by Best comments

- Ordering by highest rated

- Ordering by lowest rated

- Ordering by Reader Recommended

- Ordering by most Discussed

- Threading Models:

They are the general rules on types and size of link chains (Homogeneous or Heterogeneous). Homogeneous: all links have the same semantic: e.g. refutation. Heterogeneous: links can have different semantics: e.g. refutation, support. There are a several types available:

- one-homogeneous

- one-heterogeneous

- unlimited-homogeneous

- unlimited-heterogeneous

- limited-homogeneous

- limited-heterogeneous

Some of the study platforms are only one link and unlimited-homogeneous/replies but not all of them. As it can be observed from this comparison, most discussion forums associated with articles on these news sources seem to be designed not with the purpose of structuring arguments but mainly with the purpose of helping readers brainstorm easily their reactions to the corresponding news item.

\section{CONCLUSION}

Threading models for representing online news forums constitute a relatively unexplored field of research. This area 
significantly relates to many different activities in our lives such as communication, chatting, argumentation, and education. Here, we introduce a classification of debate threading models. We identified and classified some generic tools for collaboration used on discussion platforms on the most influent online forums. The classified tools are: Arguments, Thumbs up/down, Voting, Threading, etc. On the Internet, we can find many fora attached to some blogs and other sites that provide the users with a comment/justification opportunity. However, most of the time, they are not designed to provide or extract a conclusion on any ongoing debate, but rather to help readers brainstorm their reactions. Some web applications are nevertheless dedicated to structuring arguments with the potential intention of reaching conclusions. We here studied the relation between the features of online forums and the purpose of these forums.

While these fora utilize comments to support or negate the author's message or other comments, no thorough analysis has been performed on analyzing the features associated with forums as a function of the purpose of the forum.

\section{REFERENCES}

[1] T. Quandt, "News on the world wide web? a comparative content analysis of online news in europe and the united states," Journalism Studies, vol. 9, no. 5, pp. 717-738, 2008.

[2] F. Von Reischach, D. Guinard, F. Michahelles, and E. Fleisch, "A mobile product recommendation system interacting with tagged products," in Pervasive Computing and Communications, 2009. PerCom 2009. IEEE International Conference on. IEEE, 2009, pp. 1-6.

[3] A. Kosic and A. Triandafyllidou, "Representations of the european union and the nation (-state) in italian party discourse: A critical analysis of electoral platforms and parliamentary debates," Journal of Language and Politics, vol. 3, no. 1, pp. 53-80, 2004.

[4] L. Cotula, Land grab or development opportunity?: agricultural investment and international land deals in Africa. Iied, 2009.

[5] V. Krishnan and K. T. Ulrich, "Product development decisions: A review of the literature," Management science, vol. 47, no. 1, pp. 1-21, 2001.

[6] A. Bruns, "Stuff that matters: Slashdot and the emergence of open news," 2003.

[7] A. Alqahtani and M. Silaghi, "Classification of debate threading models for representing decentralized debates," ICAI'15 - The 17th International Conference on Artificial Intelligence, Nevada, 2015.

[8] —, "User interfaces for representing knowledge stemming from debates: Evaluating the impact of threading models (reviews) on online products," ICAI'15 - The 17th International Conference on Artificial Intelligence, Nevada, 2015.

[9] — "Evaluation the impact of human interaction/debate on online news to improve user interfaces for debate applications," Vol. 6 - Issue 1 (January - 2016), International Journal of Engineering Research and Applications (IJERA), ISSN: 2248-9622, www.ijera.com.

[10] - ,Expectations for electronic debate platforms as a function of application domains," Vol. 6 - Issue 1 (January - 2016), International Journal of Engineering Research and Applications (IJERA), ISSN: 2248-9622, www.ijera.com.

[11] "https://yourview.org.au/."

[12] M. C. Silaghi, K. Alhamed, O. Dhannoon, S. Qin, R. Vishen, R. Knowles, I. Hussien, Y. Yang, T. Matsui, M. Yokoo et al., "Directdemocracyp2pdecentralized deliberative petition drives," in Peer-to-Peer Computing (P2P), 2013 IEEE Thirteenth International Conference on. IEEE, 2013, pp. 1-2.

[13] K. Kattamuri, M. Silaghi, C. Kaner, R. Stansifer, and M. Zanker, "Supporting debates over citizen initiatives," in Proceedings of the 2005 national conference on Digital government research. Digital Government Society of North America, 2005, pp. 279-280.

[14] R. Cross, S. P. Borgatti, and A. Parker, "Making invisible work visible: Using social network analysis to support strategic collaboration," California management review, vol. 44, no. 2, pp. 25-46, 2002.
[15] A. Mukherjee and B. Liu, "Modeling review comments," in Proceedings of the 50th Annual Meeting of the Association for Computational Linguistics: Long Papers-Volume 1. Association for Computational Linguistics, 2012, pp. 320-329.

[16] M.-W. Inc., Merriam-Webster's dictionary. Merriam-Webster.

[17] C. Rinner, "Argumentation maps: Gis-based discussion support for online planning," Environment and Planning B: Planning and Design, vol. 28, no. 6, pp. 847-863, 2001.

[18] A. Funk, Y. Li, H. Saggion, K. Bontcheva, and C. Leibold, "Opinion analysis for business intelligence applications," in Proceedings of the first international workshop on Ontology-supported business intelligence. ACM, 2008, p. 3.

[19] D. C. Challener, R. A. Kelley, and P. E. Newman, "Method and system for secure computer moderated voting," Jun. 27 2000, uS Patent $6,081,793$.

[20] J. A. Halderman, H. Hursti, J. Kitcat, M. MacAlpine, T. Finkenauer, and D. Springall, "Security analysis of the estonian internet voting system," ACM, Tech. Rep., 2014.

[21] J. D. Barkan, "Comment: Further reassessment of conventional wisdom: political knowledge and voting behavior in rural kenya." American Political Science Review, vol. 70, no. 02, pp. 452-455, 1976.

[22] T. Kriplean, J. Morgan, D. Freelon, A. Borning, and L. Bennett, "Supporting reflective public thought with considerit," in Proceedings of the ACM 2012 conference on Computer Supported Cooperative Work. ACM, 2012, pp. 265-274.

[23] R. Bhathal, "Australian optical seti project," in Bioastronomy 99, vol. 213, 2000, p. 553.

[24] J. Harris and A. Henderson, "Discourse architecture," ACM SIGCHI Bulletin, vol. 30, no. 2, pp. 147-151, 1998.

[25] P. K. Gilbert and N. Dabbagh, "How to structure online discussions for meaningful discourse: A case study," British Journal of Educational Technology, vol. 36, no. 1, pp. 5-18, 2005.

[26] D. Fensel, Ontologies. Springer, 2001.

[27] M. Uschold and M. Gruninger, "Ontologies: Principles, methods and applications," The knowledge engineering review, vol. 11, no. 02, pp. 93-136, 1996.

[28] W. Clocksin and C. S. Mellish, Programming in PROLOG. Springer Science \& Business Media, 2003.

[29] S. McIlraith and T. C. Son, "Adapting golog for composition of semantic web services," KR, vol. 2, pp. 482-493, 2002.

[30] H. J. Levesque, R. Reiter, Y. Lesperance, F. Lin, and R. B. Scherl, "Golog: A logic programming language for dynamic domains," The Journal of Logic Programming, vol. 31, no. 1, pp. 59-83, 1997.

[31] W. A. Woods, "Whats in a link: Foundations for semantic networks," Representation and understanding: Studies in cognitive science, pp. 3582, 1975.

[32] P. Vossen, A multilingual database with lexical semantic networks. Springer, 1998

[33] M. J. Eppler, "A comparison between concept maps, mind maps, conceptual diagrams, and visual metaphors as complementary tools for knowledge construction and sharing," Information visualization, vol. 5, no. 3, pp. 202-210, 2006

[34] T. Buzan, "Mind maps at work: how to be the best at your job and still have time to play," 2005.

[35] G. A. Miller, "Wordnet: a lexical database for english," Communications of the ACM, vol. 38, no. 11, pp. 39-41, 1995.

[36] C. Fellbaum, WordNet. Wiley Online Library, 1998.

[37] C. Twardy, "Argument maps improve critical thinking," Teaching Philosophy, vol. 27, no. 2, pp. 95-116, 2004.

[38] P. A. Kirschner, S. J. Buckingham-Shum, and C. S. Carr, Visualizing argumentation: Software tools for collaborative and educational sensemaking. Springer Science \& Business Media, 2012.

[39] T. van Gelder, "Mapping the arguments in an academic text," 2011.

[40] H. Wache, T. Voegele, U. Visser, H. Stuckenschmidt, G. Schuster, H. Neumann, and S. Hübner, "Ontology-based integration of information-a survey of existing approaches," in IJCAI-01 workshop: ontologies and information sharing, vol. 2001. Citeseer, 2001, pp. $108-117$.

[41] M. B. Brown, "Survey article: Citizen panels and the concept of representation*," Journal of Political Philosophy, vol. 14, no. 2, pp. 203-225, 2006.

[42] A. M. McDonald, R. W. Reeder, P. G. Kelley, and L. F. Cranor, "A comparative study of online privacy policies and formats," in Privacy enhancing technologies. Springer, 2009, pp. 37-55. 
[43] J. Brank, M. Grobelnik, and D. Mladenić, "A survey of ontology evaluation techniques," 2005.

[44] T. R. Gruber, "Toward principles for the design of ontologies used for knowledge sharing?" International journal of human-computer studies, vol. 43, no. 5, pp. 907-928, 1995.

[45] J. Edelmann and B. Owsnicki, "Data models in knowledge representation systems: A case study," in GWAI-86 und 2. Österreichische Artificial-Intelligence-Tagung. Springer, 1986, pp. 69-74.

[46] H. R. Kim and P. K. Chan, "Learning implicit user interest hierarchy for context in personalization," in Proceedings of the 8th international conference on Intelligent user interfaces. ACM, 2003, pp. 101-108.

[47] J. J. Cimino, P. D. Clayton, G. Hripcsak, and S. B. Johnson, "Knowledge-based approaches to the maintenance of a large controlled medical terminology." Journal of the American Medical Informatics Association, vol. 1, no. 1, p. 35, 1994.

[48] D. G. Bobrow, G. G. Hendrix, W. A. Martin, J. McCarthy, A. Newell, R. Schank, B. C. Smith, and N. Sridharan, "A panel on knowledge representation," in Proceedings of the 5th international joint conference on Artificial intelligence-Volume 2. Morgan Kaufmann Publishers Inc., 1977, pp. 983-992.

[49] T. K. Landauer and S. T. Dumais, "A solution to plato's problem: The latent semantic analysis theory of acquisition, induction, and representation of knowledge." Psychological review, vol. 104, no. 2, p. 211, 1997.

[50] M. Zhou, Y. J. Son, and Z. Chen, "Knowledge representation for conceptual simulation modeling," in Proceedings of the 36th conference on Winter simulation. Winter Simulation Conference, 2004, pp. 450458.

[51] J. F. Sowa, "Knowledge representation: logical, philosophical, and computational foundations," 1999.

[52] U. Fayyad, G. Piatetsky-Shapiro, and P. Smyth, "From data mining to knowledge discovery in databases," AI magazine, vol. 17, no. 3, p. 37, 1996.

[53] C. Reed and G. Rowe, "Araucaria: Software for argument analysis, diagramming and representation," International Journal on Artificial Intelligence Tools, vol. 13, no. 04, pp. 961-979, 2004.

[54] G. Fischer, A. C. Lemke, R. McCall, and A. I. Morch, "Making argumentation serve design," Human-Computer Interaction, vol. 6, no. 3-4, pp. 393-419, 1991.

[55] K. Subrahmanyam, S. M. Reich, N. Waechter, and G. Espinoza, "Online and offline social networks: Use of social networking sites by emerging adults," Journal of Applied Developmental Psychology, vol. 29, no. 6, pp. 420-433, 2008.

[56] T. J. Berners-Lee, "The world-wide web," Computer Networks and ISDN Systems, vol. 25, no. 4, pp. 454-459, 1992.

[57] D. Hansen, B. Shneiderman, and M. A. Smith, Analyzing social media networks with NodeXL: Insights from a connected world. Morgan Kaufmann, 2010.

[58] M. E. Newman, "Communities, modules and large-scale structure in networks," Nature Physics, vol. 8, no. 1, pp. 25-31, 2012.

[59] M. Crispin and K. Murchison, "Internet message access protocol-sort and thread extensions," RFC 5256, June, Tech. Rep., 2008.

[60] S. J. Delany, P. Cunningham, and B. Smyth, "Ecue: A spam filter that uses machine learning to track concept drift," 2006.

[61] "http://searchengineland.com/revealing-the-sources-of-google-news11353," Revealing The Sources Of Google News, 2007.

[62] H. M. Robert, Pocket Manual of Rules of Order for Deliberative Assemblies. SC Griggs, 1896. 